\title{
Presentación al Dossier Geografía, política y resistencias: perspectivas, casos y estrategias de abordaje
}

Mariana Arzeno

CONICET - Universidad de Buenos Aires. Facultad de Filosofía y Letras. Instituto de Geografía "Romualdo Ardissone". Buenos Aires, Argentina.

\section{Mónica Farías}

CONICET - Universidad de Buenos Aires. Facultad de Filosofía y Letras. Instituto de Geografía "Romualdo Ardissone". Buenos Aires, Argentina.

\author{
Sam Halvorsen \\ School of Geography. Queen Mary University of London. Reino Unido.
}

\section{Introducción}

El presente dossier es resultado de una serie de colaboraciones e intercambios entre investigadores del Grupo de Estudios Geografías Emergentes del Instituto de Geografía de la UBA (GEm-IIGEO) y de la red de trabajo Latin American Geographies in the United Kingdom (LAG-UK), que tuvieron un hito importante en 2018 a partir de la organización de la Mesa Redonda "Geografía y Política en América Latina: espacialidad de y en los procesos políticos". Dicha Mesa reunió a investigadores de distintas unidades académicas de Latinoamérica y también del Reino Unido, así como a militantes de organizaciones sociales, interesados en debatir respecto de dos interrogantes centrales: ¿qué papel le compete a la geografía en la política de América Latina en la actualidad? ¿Cómo contribuye la disciplina a pensar lo político tanto en su dimensión teórica como en la materialidad de las prácticas cotidianas? Las discusiones giraron en torno a los procesos políticos de resistencias y conflictos que vienen cobrando visibilidad en las últimas décadas en América Latina, en particular aquellos que surgen en torno a tensiones entre actores por la apropiación del espacio y organización de la vida socioespacial. La organización del dossier constituyó una instancia para materializar algunas de las discusiones que se plantearon durante este evento -así como para sumar otras nuevas - con el objetivo de profundizar la discusión teórica y epistemológica relativa a la espacialidad de esos procesos políticos, en cuanto elemento indisociable de los mismos. Otro de los objetivos ha sido el de promover el diálogo entre geógrafxs y otras disciplinas en lo que respecta a su estudio.

En las últimas décadas en Argentina, tal como en otros países de la región, hubo un notorio giro espacial en torno al análisis de los procesos de resistencias frente al avance y la consolidación de políticas y prácticas - por parte de privados y también del Estado- excluyentes, desiguales y hasta violentas. Esto se evidencia, por ejemplo, en 
el rol central que ha ocupado el territorio en múltiples trabajos sobre la movilización colectiva, en donde se subraya ya sea la importancia de las identidades, los vínculos sociales y los valores producidos en los barrios urbanos (Grimson, Ferraudi y Segura, 2009; Manzano, 2013; Merklen, 2005; Rodríguez, 2018; Rodríguez y Di Virgilio, 2016; Svampa y Pereyra, 2009, Rossi, 2017), así como las experiencias comunitarias en zonas extractivas y del agronegocio (Domínguez, 2009, 2016; Svampa, 2011; Wahren, 2012). Desde el campo de la geografía algunos trabajos se han centrado específicamente en la dimensión espacial de las movilizaciones colectivas. Por ejemplo, los trabajos de Tobío (2011; 2014) sobre la territorialidad piquetera, los de Sznol (2007) sobre la espacialidad de la protesta social en ámbitos urbanos y Pintos (2004) sobre la espacialidad de las resistencias de movimientos sociales.

En este dossier nos proponemos, en primer lugar, subrayar la importancia de seguir pensando el giro espacial en clave geográfica, es decir, desde las orientaciones epistemológicas y con las herramientas conceptuales y metodológicas del campo disciplinar geográfico. En segundo lugar, buscamos llamar la atención acerca de la relevancia de conceptos como el de "lugar" o ideas como "ordenamiento espacial" que no son utilizados con tanta frecuencia como el de "territorio" para dar cuenta de los procesos políticos. Por último, consideramos necesario destacar la variedad de actores sociales en los procesos políticos de resistencia, actores a los que frecuentemente no se los ve actuando e incidiendo en el plano de lo político. Por ello, este dossier busca interrogar y problematizar el recurso del espacio y la constelación de conceptos asociados a él (Haesbaert, 2014) que abundan en el lenguaje cotidiano de las ciencias sociales en la actualidad. Consideramos necesario fomentar un diálogo que examine la importancia de la geografía y el de un enfoque geográfico sobre los fenómenos políticos, que también avance en la discusión teórica sobre el espacio, el territorio y el lugar como conceptos nodales para problematizar la dimensión espacial de los procesos políticos de resistencias.

Los seis artículos que conforman este dossier presentan discusiones que no sólo buscan problematizar la dimensión espacial implicada en los procesos que analizan, sino que además proponen, en algunos casos, temas poco trabajados en general desde el campo disciplinar. Cada uno de ellos aporta elementos teóricos y empíricos sobre procesos de resistencia, algunos de los cuales son protagonizados por movimientos sociales/ movimientos socioterritoriales (en el caso de Torres y Halvorsen) y, en otros casos, por actores que no se definen como movimiento, pero que sin embargo presentan grados diversos de organización y/o articulación entre sí y con el Estado (son los casos de Fernández et al., Jurado, Cardozo y Palladino). En estos trabajos el espacio y otras categorías espaciales se vuelven relevantes para comprender la diversidad y complejidad de los procesos de resistencia en el contexto actual, así como para pensar estrategias de lucha y de cambio social. De este modo, lxs autores contribuyen a profundizar la discusión teórica relativa a la espacialidad de (su expresión espacial), así como también la espacialidad en los procesos (en tanto elemento co-constitutivo y por lo tanto productor de estos). Para su abordaje recurren a diversas herramientas analíticas -espacio, orden espacial, territorio, territorialización, lugar- que ponen en juego en el análisis empírico a través de distintas formas de operacionalización.

El dossier comienza con el artículo de Fernández Romero, Muñecas, Zanotti y Piccinali, Mapeando el (des)orden espacial: cartografía social en Cabure-í, Misiones. Allí lxs 
autores exploran los modos en que la cartografía social puede contribuir a poner de relieve la dimensión espacial en las situaciones donde existen tensiones en torno al uso, ocupación o apropiación del espacio. El artículo analiza una práctica de mapeo colectivo que llevaron a cabo con una cooperativa campesina del norte de la provincia de Misiones en conflicto con los titulares registrales de las tierras que habitan. Recuperando discusiones recientes del campo de las cartografías críticas, esta práctica de mapeo les permitió problematizar las ideas de orden/desorden espacial, así como el rol de las cartografías oficiales en la producción/contestación del ordenamiento espacial. El mapeo colectivo resultó en un mapa en donde la distribución de las tierras ocupadas por las familias respeta los límites perimetrales del loteo del catastro, aunque los usos y apropiaciones son diferentes a los prescriptos. Asumiendo al ordenamiento como una práctica intrínseca a la producción del espacio, en donde la definición de "orden" está siempre en disputa, este caso demuestra que las cartografías oficiales pueden ser apropiadas, interpretadas y usadas creativamente de múltiples formas como parte de órdenes espaciales alternativos.

De alguna manera, este artículo deja planteado el interrogante sobre qué sujetos se constituyen como actores políticos y cómo la politicidad de las acciones queda en evidencia cuando recurrimos a un análisis espacial. Un tema que se repite en los tres siguientes artículos. En este sentido, los dos primeros se adentran en discusiones sobre la economía social y solidaria (ESS), un campo de estudios académicos y de debate político que desde hace dos décadas viene cobrando relevancia en Argentina. En su artículo Territorio y lugar: la espacialidad en debate en la Economía Social y Solidaria, Jurado recupera algunas de las principales discusiones teóricas sobre los conceptos "territorio" y "lugar", y hace un ejercicio analítico para abordar con cada concepto el caso de una organización campesina de la ESS en Mendoza. El autor reafirma la pertinencia del concepto de territorio en el abordaje de las luchas contra los cercamientos y la integración a la lógica del capital de espacios comunitarios por parte de productores campesinos. Sin embargo, también encuentra limitaciones en la capacidad de dar cuenta de otras dimensiones. Así, recurre al concepto de lugar para analizar el entramado de acciones que desarrollan estas experiencias una vez que lograron territorializarse (es decir, controlar un área geográfica que deviene espacio apropiado por la organización, donde llevan adelante sus proyectos productivos). Según el autor, la concepción de lugar que articula las dimensiones de localización o emplazamiento (y su relación con otros lugares), locale (o escenas donde las actividades diarias se desarrollan) y sentido de lugar, resulta muy productiva para explicar desde una perspectiva geográfica el origen y despliegue de circuitos alternativos en contextos de resistencia campesina.

Por su parte, Cardozo, en su artículo Políticas de promoción de la Economía Social y Solidaria en la comunidad mocoví Com-Caia de Recreo (Santa Fe, Argentina). La construcción de circuitos cortos de comercialización en el período 2012-2017, focaliza en la territorialización de las políticas para ESS en esa comunidad. El autor recupera algunas discusiones que permiten conceptualizar la dimensión territorial de la implementación de las políticas públicas. Para ello, retomando el planteo de Haesbaert (2018), pone en diálogo la idea de territorio como categoría normativa que enfatiza la mirada estatal sobre el mismo, con la idea del territorio como categoría de la práctica política de los sectores subalternos que son objeto de esas políticas. Este cruce le permite analizar el "encuentro" entre las formas de territorialización de ciertos procesos espaciales que atraviesa la comunidad y las intervenciones de distintos 
actores con proyectos que involucran a esta población. Así, la territorialización de la política se manifiesta en nuevas estrategias de organización socioespacial que emergen de la negociación entre los actores. Esto implica negociar las condiciones de posibilidad para la puesta en funcionamiento de circuitos cortos de comercialización campo-ciudad de productos hortícolas. Cardozo, además, se detiene y reflexiona en algunos de los procesos por los que atraviesa una comunidad indígena que logra el acceso a la tierra y que además avanza en el desarrollo de procesos productivos para su reproducción. Se trata, en definitiva, de comunidades cuya adscripción territorial es producto de un complejo proceso de desplazamientos y de luchas, algo que es retomado en el siguiente artículo.

El trabajo de Palladino, Territorio(s) e indigeneidad(es): aportes a partir del trabajo de campo con comunidades comechingonas cordobesas, focaliza su análisis en la forma en que los procesos de re-emergencia comunal de los comechingones en la provincia de Córdoba construye la adscripción territorial apelando a distintas formas de identificación con el territorio. Para ello el autor toma dos casos de estudio en donde discute el papel del territorio en los procesos de comunalización desde la perspectiva relacional e integradora propuesta por el geógrafo Rogerio Haesbaert, lo que le permite abordar los procesos de territorialización indígena desde la movilidad e identificación territorial con distintos sitios. Así, el autor se distancia de los enfoques que identifican las reivindicaciones indígenas con territorialidades sustentadas en la continuidad espacial, es decir,en la fijación y la rigidez en lo que respecta al vínculo identidad-territorio. Esto da lugar a la posibilidad de problematizar dichas territorialidades como móviles y fluidas, y asociadas a los procesos de desagregación y fractura a las que fueron sometidas durante la colonia. El autor muestra cómo los procesos de des-territorialización y re-territorialización se vuelven elementos clave en la reconstrucción de narrativas y memoria territorial en la que se basan esos procesos de reemergencias y comunalización.

Finalmente, cierran el dossier dos artículos que ponen el foco en los movimientos socioterritoriales, aportando distintas discusiones que permiten, por un lado, operacionalizar el concepto de manera tal de abordar la dimensión espacial intrínseca a ellos y, por otro, complejizar el análisis relativo a sus vínculos con el Estado en la producción del territorio de los movimientos. En el artículo Movimientos sociales e institucionalización: la especificidad de los movimientos socioterritoriales, Torres presenta un método analítico para entender las relaciones entre movimientos sociales e instituciones, recurriendo a la categoría de movimiento socioterritorial y recuperando las discusiones teóricas planteadas por Fernandes en torno a sus particularidades (2005). La autora se centra en la dimensión de la institucionalización de las formas organizacionales, en y a través del territorio, característica de los movimientos (Halvorsen, Fernandes y Torres, 2019); es decir, la producción de instituciones que garanticen las relaciones de poder que sustentan esa apropiación del espacio convertido en el territorio del movimiento. Para ello utiliza el caso de la Organización Barrial Túpac Amaru de Jujuy, que por medio de la ocupación de tierras logró construir un barrio y producir instituciones laborales, educativas, de salud y culturales, entre otras. El control del territorio habilita relaciones sociales, reglas y prácticas que regulan la vida cotidiana desde lógicas distintas a la mercantil. De este modo, para la autora, los movimientos socioterritoriales dejan en evidencia la importancia de la dimensión espacial, específicamente la producción y control de territorios para la creación de instituciones y nuevas ciudadanías territorializadas. 
Halvorsen también recurre al concepto de movimiento socioterritorial en su artículo El Territorio en disputa: estrategias políticas y movimientos socioterritoriales, para discutir el concepto "territorio". El autor pone en diálogo las discusiones anglosajonas sobre el territorio -como el ámbito de ejercicio del poder estatal-y las latinoamericanas -en donde abundan las referencias al territorio como ámbito de poder emancipatorio para los movimientos-. Apoyándose en tres tipos de movimiento de América Latina cuyo objetivo es el de la apropiación de un espacio determinado, Halvorsen sugiere que lejos de ser algo estable, el territorio es un proceso de apropiación siempre aconteciendo. Propone entonces una definición abierta de territorio que resulta de la apropiación del espacio a través de estrategias políticas intrincadas y superpuestas. Esto supone considerar al menos tres dimensiones: las configuraciones territoriales hegemónicas, pasadas y presentes, en donde tiene un rol predominante el Estado; la coexistencia de diversas apropiaciones que generan múltiples territorios; y el cruce de diversas prácticas e ideas que materializan relaciones de poder en el espacio. El autor recupera estas dimensiones en el análisis de los casos para detenerse en las distintas negociaciones y puntos de tensión entre éstos y las estrategias de control del Estado. Esto le permite alejarse de las miradas romantizadas sobre los movimientos que enfatizan mayormente su poder emancipatorio, al tiempo que deja en evidencia la potencialidad de los cruces epistemológicos provenientes de distintas tradiciones académicas.

En su conjunto el dossier sugiere algunas posibles líneas de trabajo para continuar con el debate sobre la espacialidad de los procesos políticos y el potencial de la geografía para dar cuenta de ellos. Creemos necesario continuar y extender las discusiones acerca de la/s espacialidad/es de las resistencias presentes en la región. Dado el fuerte interés que el concepto de territorio ha suscitado en las ciencias sociales en América Latina en los últimos años -al punto de plantearse un "giro territorial" más que espacial en la región (Haesbaert, 2009; Souza, 2009)-, consideramos particularmente importante ahondar en otras espacialidades de la política y en los modos en los que las mismas se relacionan. Pensar los procesos políticos a partir de los conceptos de lugar, escala, red, paisaje (ej. Jessop et al., 2008) no sólo permite enriquecer su discusión teórica, sino que además habilita nuevos campos de acción para la práctica política. Además, creemos necesario dar lugar a más y mayores diálogos interdisciplinarios en lo que respecta a la producción de saberes sobre la espacialidad de y en los procesos políticos. Para ello será necesario tener presente la construcción de los bordes disciplinares y las "regiones ontológicas" (Lander, 2000) de las disciplinas implicadas en el "giro espacial”, de modo de alentar su constante revisión y enriquecer así el intercambio. Por último, resultaría interesante considerar la importancia de los lugares y los ámbitos donde estos saberes emergen, se manifiestan y circulan. Tener en cuenta la situacionalidad de los saberes anclados en contextos geohistóricos específicos -Argentina, América Latina- necesariamente requeriría repensar los modos en los que se traducen y comunican esos saberes.

La foto de la portada fue tomada en el año 2016 en la localidad de Tilcara, situada en la Quebrada de Humahuaca, provincia de Jujuy. Se trata de un graffiti que expresa una serie de resistencias que se vienen dando en ese lugar y en la Quebrada en general: resistencias a la minería a cielo abierto, de uranio y otros minerales, resistencias a la valorización inmobiliaria que se deriva del turismo. Como dijo Rodolfo Walsh, las paredes son la imprenta de los pueblos, de aquellxs que, sin tener el poder para llegar a los grandes medios de comunicación, buscan modos de comunicarse, de hacer oír su voz 
Presentación al Dossier Geografía, política y resistencias: perspectivas, casos y estrategias de abordaje

MARIANA ARZENO, MónICA FARÍAS, SAM HALVORSEN

y visibilizar sus reclamos. Podemos pensar a este graffiti como una práctica espacial de resistencia (Souza, 2013), un modo de visibilizar y rectificar una situación de injusticia socioespacial. Nos pareció entonces apropiado, sumar esta imagen a las geografías de las resistencias presentes en este dossier. 


\section{Q Bibliografía}

"Domínguez, D. (2009). La lucha por la tierra en Argentina en los albores del Siglo XXI. La recreación del campesinado y de los pueblos originarios. Tesis de doctorado, Facultad de Ciencias Sociales, Universidad de Buenos Aires.

»Domínguez, D. (2016). Territorialidades campesinas entre lo heterónomo y lo disidente: formas de gestión de la producción y tenencia de la tierra en el campo argentino. Política \& Trabalho. Revista de Ciências Sociais, 45, 67-84.

"Fernandes, B. M. (2005). Movimentos Socioterritoriais e Movimentos Socioespaciais: Contribuição Teorica Para Uma Leitura Geografica Dos Movimentos Sociais. Revista Nera, 8(6), 14-34.

» Grimson, A., Ferraudi, C. y Segura, R. (Eds) (2009). La vida política en los barrios populares de Buenos Aires. Buenos Aires: Ed. Prometeo.

»Haesbaert, R. (2009). Dilema de conceitos: espaço-território e contenção territorial. En M. A., Saquet y E. Sposito (Org.), Territórios e territorialidades: teorias, processos e conflitos (pp. 95-120). São Paulo: Expressão Popular.

" Haesbaert, R. (2014). Por uma constelação de conceitos. En R. Haesbaert, Viver no limite. Território e multi/transterritorialidade em tempos de in-segurança e contenção (pp. 19-51). Rio de Janeiro: Bertrand Brasil.

» Haesbaert, R. (2018). De categoria de análise a categoria da prática: A multiplicidade do território numa perspectiva latino-americana. En F., Fridman, L., Alem Gennari y S. Lencioni (Org.). Políticas públicas e territórios: onze estudos latinoamericanos (pp. 267-288). Ciudad Autónoma de Buenos Aires: CLACSO.

» Halvorsen, S. (2018). Cartographies of epistemic expropriation: critical reflections on learning from the South. Geoforum, 95, 11-20.

» Halvorsen, S. Fernandes, B. M. y Torres, F. (2019). Mobilising Territory: Socioterritorial movements in comparative perspective. Annals of the Association of American Geographers, 109(5), 1454-1470.

» Jessop, B., Brenner, N., y Jones, M. (2008). Theorizing sociospatial relations. Environment and Planning D: Society and Space, 26, 389-401. https://doi. org/10.1068/d9107

"Lander, E. (2000). La colonialidad del saber: eurocentrismo y ciencias sociales: perspectivas latinoamericanas. Caracas, Venezuela: Facultad de Ciencias Económicas y Sociales (FACES-UCV): Instituto Internacional de la UNESCO para la Educación Superior en América Latina y el Caribe (IESALC).

" Manzano, V (2013). La política en movimiento: Movilizaciones colectivas y políticas estatales en la vida del Gran Buenos Aires. Rosario: Prohistoria ediciones.

» Merklen, D. (2005). Pobres ciudadanos: Las clases populares en la era democrática (Argentina 1983-2003). Buenos Aires: Editorial Gorla.

»Pintos, P. (2004). La espacialidad de la resistencia social: entre la visibilidad en las calles y la acción en el territorio. Notas sobre la espacialidad piquetera en la Argentina reciente. Reflexiones geográficas, 11, 45-67. 
》Rodríguez, M. C. (2018). Género, espacialidad y urbanismo autogestionario. Algunas claves para su comprensión y debate. Revista Vivienda y Ciudad, 5, 67-79.

» Rodríguez, M. C. y Di Virgilio, M. (2016). A city for all? Public policy and resistance to gentrification in the southern neighborhoods of Buenos Aires. Urban Geography, $37,1215-1234$.

» Rossi, F. (2017). The Poor's Struggle for Political Incorporation: The Piquetero Movement in Argentina. Cambridge: Cambridge University Press.

»Souza, M. L. de (2013). Os conceitos fundamentais da pesquisa sócio-espacial. Rio de Janeiro: Bertrand Brasil.

»Souza, M. L. de (2009). Território da divergência (e da confusão): Em torno das imprecisas fronteiras de um conceito fundamental. En M. A. Saquet y E. S. Sposito (Org.) Territórios e Territorialidades: Teorias, processos e conflitos (pp. 57-72). São Paulo: Expressão Popular.

»Svampa, M., y Pereyra, S. (2009). Entre la ruta y el barrio: La experiencia de las organizaciones piqueteras. Buenos Aires: Editorial Biblos.

»Sznol, F. (2007). Geografía de la Resistencia. Protesta social, formas de apropiación y transformación del espacio urbano en la Argentina (1996-2006). Revista Theomai, 15, 21-34.

" Tobío, O. (2011). Entre la acción territorial directa y las "piedras de papel": Movimientos sociales y modelo de desarrollo en la Argentina. Revista Geográfica de América Central, 2(47E), 1-11.

»Tobío, O. (2014) El doble carácter de la territorialidad de la experiencia piquetera en el norte de la Argentina: una conceptualización. XIII Coloquio Internacional de Geocrítica El control del espacio y los espacios de control, Barcelona.

"Wahrren, J. (2012). Movimientos Sociales, y territorios en disputa. Experiencias de trabajo y autonomía de la Unión de Trabajadores Desocupados de Gral. Mosconi, Salta. Trabajo y Sociedad, 19, 133-147. 